\title{
Type 1 Diabetes
}

\author{
Francesco Chiarelli and Cosimo Giannini \\ Department of Pediatrics, University of Chieti, Chieti, Italy
}

\begin{abstract}
It is well known that type 1 diabetes is a chronic autoimmune disease in which the breakdown of normal tolerance to the $\beta$ cell allows the activation of $T$ cell to multiple antigens. This induces a progressive destruction of $\beta$ cells and a state of chronic insulin deficiency. Multiple genetic and environmental risk factors have been evaluated for better understanding the mechanisms causing type 1 diabetes with the aim of improving prediction and possibly prevention of $\beta$ cell impairment and failure. Meanwhile, it remains of paramount importance to reach the best metabolic and glycemic control from the very beginning of diabetes in children and adolescents; new technologies have improved the day-to-day diabetes care and there is hope that a closed-loop device will allow a more physiological insulin delivery. There is also hope for finding a cure for diabetes by teaching stem cells to produce insulin, by implementing $\beta$ cell and pancreas transplantation and by applying gene therapy.
\end{abstract}

\section{Mechanism of the year}

\section{Kv1.3 channels are a therapeutic target for T-cell-mediated autoimmune diseases}

Beeton C, Wulff H, Standifer NE, Azam P, Mullen KM, Pennington MW, Kolski-Andreaco A, Wei E, Grino A, Counts DR, Wang PH, LeeHealey CJ, Andrews BS, Sankaranarayanan A, Homerick D, Roeck WW, Tehranzadeh J, Stanhope KL, Zimin P, Havel PJ, Griffey S, Knaus HG, Nepom GT, Gutman GA, Calabresi PA, Chandy KG

Department of Physiology and Biophysics, University of California, Irvine, Calif., USA

gchandy@uci.edu

Proc Natl Acad Sci USA 2006;103:17414-17419

Background: Autoreactive memory $\mathrm{T}$ lymphocytes are implicated in the pathogenesis of autoimmune diseases.

Methods and Results: Here the authors demonstrate that disease-associated autoreactive T cells from patients with type 1 diabetes mellitus or rheumatoid arthritis are mainly CD4+ CCR7-CD45RA-effector memory $\mathrm{T}$ cells with elevated Kv1.3 potassium channel expression. In contrast, T cells with other antigen specificities from these patients, or autoreactive $\mathrm{T}$ cells from healthy individuals and disease controls, express low levels of Kv1.3 and are predominantly naive or central-memory cells. In effector memory T cells, Kv1.3 traffics to the immunological synapse during antigen presentation where it colocalizes with Kvß2, SAP97, ZIP, p56(lck), and CD4. Although Kv1.3 inhibitors do not prevent immunological synapse formation, they suppress $\mathrm{CA}^{2+}$ signaling, cytokine production, and proliferation of autoantigenspecific effector memory $\mathrm{T}$ cells at pharmacologically relevant concentrations while sparing other classes of T cells. Kv1.3 inhibitors ameliorate pristane-induced arthritis in rats and reduce the incidence of experimental autoimmune diabetes in diabetes-prone BB rats. Repeated dosing with Kv1.3 inhibitors in rats has not revealed systemic toxicity.

Conclusions: Further development of Kv1.3 blockers for autoimmune disease therapy is warranted.

Type 1 diabetes is an autoimmune disease characterized by a permissive immune system that fails to impose tolerance to arrays of self-antigens [1]. Therefore, disease-modifying immunotherapies capable of selectively suppressing autoantigens reaction are under investigation. Because the diseaseassociated autoreactive T cells are mainly costimulation-independent by CCR7-effector memory T cells, immunomodulators able to selectively suppress effector memory $T$ cells without affecting other lymphoid subsets would be relevant [2]. This study evaluated disease-associated autoreactive T cells from patients with type 1 diabetes or rheumatoid arthritis and tested whether a selective blockers of Kv1.3 (a homotetrameric human $\mathrm{K}^{+}$channel which regulates membrane potential and $\mathrm{Ca}^{2+}$ signalling in 
human T cells, overexpressed in activated CD4+ and CD8+ effector memory T/effector memory ${ }_{C D 45 R A+}$ $T$ cells) could reduce autoimmune-mediated disease in rat models of type 1 diabetes or rheumatoid arthritis, without causing toxicity. It was shown that disease-associated autoreactive T cells in type 1 diabetes, multiple sclerosis and rheumatoid arthritis are mainly CCR7-Kv1.3 $3^{\text {high }}$ effector memory $T$ cells. Furthermore, utilizing a selective Kv1.3 blocker to discern whether effector memory T-cell function can be preferentially suppressed without impacting naive/T central memory cells in rheumatoid arthritis and type 1 diabetes patients, it was observed that Kv1.3 blockers might globally suppress effector memory T/effector memory ${ }_{\mathrm{CD} 45 \mathrm{RA}} \mathrm{T}$ cells and compromise the ability to respond to pathogens. Nonetheless, conducting a prevention trial of PAP1 (a small-molecule Kv1.3 inhibitor) in $\mathrm{BB}$ rats (a standard model for type 1 diabetes), it was also demonstrated that Kv1.3 inhibitors reduce the incidence of autoimmune diabetes without relevant side effects. These encouraging data provide a rationale for evaluating Kv1.3 inhibitors as a therapy for type 1 diabetes and for preventing autoimmune destruction of HLA-matched grafted islets. Highly selective immune system modulation represents an innovating and promising therapeutic opportunity which would be ideal in prevention of type 1 diabetes.

\title{
New paradigms
}

\section{Intrahepatic transplanted islets in humans secrete insulin in a coordinate pulsatile manner directly into the liver}

\author{
Meier JJ, Hong-McAtee I, Galasso R, Veldhuis JD, Moran A, Hering BJ, Butler PC \\ Larry Hillblom Islet Research Center, University of California, Los Angeles David Geffen School of Medicine, \\ Los Angeles, Calif., USA
}

pbutler@mednet.ucla.edu

Diabetes 2006:55:2324-2332

Background: Intrahepatic islet transplantation is an experimental therapy for type 1 diabetes.

Methods: In the present studies, the authors sought to address the following questions: (1) in humans, do intrahepatic transplanted islets re-establish coordinated pulsatile insulin secretion? and (2) to what extent is insulin secreted by intrahepatic transplanted islets delivered to the hepatic sinusoids (therefore effectively restoring a portal mode of insulin delivery) versus delivered to the hepatic central vein (therefore effectively providing a systemic form of insulin delivery)? To address the first question, the authors examined insulin concentration profiles in the overnight fasting state and during a hyperglycemic clamp (ca. $150 \mathrm{mg} / \mathrm{dl}$ ) in 10 recipients of islet transplants and 10 control subjects. To address the second question, the authors measured first-pass hepatic insulin clearance in two recipients of islet autografts after pancreatectomy for pancreatitis versus 5 control subjects by direct catheterization of the hepatic vein. Results: The authors report that coordinate pulsatile insulin secretion is re-established in islet transplant recipients and that glucose-mediated stimulation of insulin secretion is accomplished by amplification of insulin pulse mass. Direct hepatic catheterization studies revealed that intrahepatic islets in humans do deliver insulin directly to the hepatic sinusoid because approximately $80 \%$ of the insulin is extracted during first pass.

Conclusions: In conclusion, intrahepatic islet transplantation effectively restores the liver to pulsatile insulin delivery.

$\beta$-Cell replacement is an attractive potential treatment in order to mimic as close as possible the insulin variation in all physiological and pathological conditions and to avoid any disadvantage related to insulin replacement. Performed in conjunction with kidney transplants or as solitary grafts, $\beta$-cell transplantation can be either a whole organ pancreas transplant or an islet cell transplant [3]. However, due to the related surgical morbidities and long-term mortality, islet cell transplantation represents a suitable procedure which offers several advantages. In particular, by secreting insulin directly into the portal-venous circulation to the liver, intrahepatic transplanted cells might reproduce the physiological variations of insulin secretion. In general, whole organ transplants have a more sustained and durable function and are more widely available than their counterparts, but 
entail a much more invasive procedure. In this study, 10 patients with type 1 diabetes were studied after islet transplantation in order to evaluate whether intrahepatic transplanted islets in humans may serve as a reliable pacemaker for pulsatile insulin secretion in a coordinated pulsatile manner. Thus, pulsatile insulin secretion, determined by deconvolution analysis of 1-minute insulin concentrations, was measured in the basal fasting state and during a hyperglycemic clamp followed by an acute intravenous arginine $(5 \mathrm{~g})$ bolus. It was shown that insulin secretion in islet transplant recipients is pulsatile and glucose-induced insulin secretion is accomplished through amplification of pulse size. Furthermore, by studying insulin concentrations in samples obtained simultaneously from the hepatic vein and the arterialized hand vein in 1-minute intervals over $40 \mathrm{~min}$, it was shown a similar hepatic first-pass insulin extraction in intraportal islet autotransplanted patients compared with healthy control subjects. These results imply a direct insulin delivery into hepatic sinusoids rather than into the hepatic central vein. This appears to be an interesting approach which should re-establish a pulsatile pattern of insulin release in patients with type 1 diabetes through intraportal islet transplantation which is able mimic both the physiological $80 \%$ first passage insulin clearance and the balance between hepatic and extrahepatic insulin exposure. However, no long-term related effect can be drawn by this study in order to evaluate the lifelong efficacy of the intraportal islet transplantation. Although promising, the surgical- and method-related consequences such as thrombosis, bleeding, and exposure of the islets to high gut-derived environmental toxins and immunosuppressive drugs used to prevent rejection, reduce drastically the utility of this procedure in the short-term. However, the well-reproduced physiological route of intraportal insulin delivery stimulates further studies as well as new pharmacological approaches in this field.

\section{New hope}

\section{Production of pancreatic hormone-expressing endocrine cells from human embryonic stem cells}

D'Amour KA, Bang AG, Eliazer S, Kelly OG, Agulnick AD, Smart NG, Moorman MA, Kroon E, Carpenter MK, Baetge EE

Novocell Inc., San Diego, Calif., USA

gage@salk.edu

Nat Biotechnol 2006;24:1392-1401

Background: Of paramount importance for the development of cell therapies to treat diabetes is the production of sufficient numbers of pancreatic endocrine cells that function similarly to primary islets. Methods and Results: the authors have developed a differentiation process that converts human embryonic stem cells to endocrine cells capable of synthesizing the pancreatic hormones insulin, glucagon, somatostatin, pancreatic polypeptide and ghrelin. This process mimics in vivo pancreatic organogenesis by directing cells through stages resembling definitive endoderm, gut-tube endoderm, pancreatic endoderm and endocrine precursor - en route to cells that express endocrine hormones. The human embryonic stem cell-derived insulin-expressing cells have an insulin content approaching that of adult islets. Similar to fetal $\beta$ cells, they release C-peptide in response to multiple secretory stimuli, but only minimally to glucose.

Conclusions: Production of these human embryonic stem cell-derived endocrine cells may represent a critical step in the development of a renewable source of cells for diabetes cell therapy.

During the gastrulation stage of embryogenesis the definitive endoderm germ layer, which is generated in an area termed the primitive streak, progressively proliferates and differentiates into different gastrointestinal cells including the endocrine pancreatic cells. A complex and strict step-by-step activation of several transcription factor genes and signals from the adjacent growing tissue are necessary to allow a complete and precise production of the different insular cells [4]. The unlimited replicative capacity and the ability to produce most, if not all, differentiated cell types allow to retain the human embryonic stem cells, a potential and interesting approach in type 1 diabetes [5]. In this study characterizing the differentiation process at the RNA and protein levels using real-time PCR, 
Western blotting, immunofluorescence and flow cytometry, it was shown that definitive endoderm derived from human embryonic stem cells can be efficiently differentiated to hormone-expressing endocrine cells. The authors reproduced a five-stage protocol for differentiating human embryonic stem cells to endocrine hormone-expressing cells through a series of endodermal intermediates resembling those that occur during pancreatic development in vivo. Furthermore, insulin content of the insulin-expressing cells approached that of adult islets. In addition, C-peptide release occurred in response to multiple secretory stimuli. However, the insulin-expressing cells' response to glucose was less effective than to what occurs in the fetal $\beta$-cell. Although endocrine hormone-expressing cells were well reproduced, it was not defined whether a differentiation process occurs via an inductive or a selective mechanism. These interesting issues need to be addressed in future studies in order to completely explore the $\beta$-cell organogenesis. This model might represent a promising way to restore a renewable source of pancreatic $\beta$ cells.

\title{
Preparation and in vitro and in vivo characterization of composite microcapsules for cell encapsulation
}

\author{
Blasi P, Giovagnoli S, Schoubben A, Ricci M, Rossi C, Luca G, Basta G, Calafiore R \\ Department of Chemistry and Technology of Drugs, School of Pharmacy, University of Perugia, Perugia, Italy \\ kaolino@unipg.it
}

Int J Pharm 2006;324:27-36

Background: Cell encapsulation technology raises great hopes in medicine and biotechnology. Transplantation of encapsulated pancreatic islets represents a promising approach to the final cure of type 1 diabetes mellitus. Unfortunately, long-term graft survival and functional competence remain only partially fulfilled. Failure was often ascribed to the lack of biocompatibility generating inflammatory response, limited immunobarrier competence, hypoxia, and low $\beta$-cell replication.

Methods: In the present work, ketoprofen-loaded biodegradable microspheres, embedded into alginate/ poly-L-ornithine/alginate microcapsules, were prepared in order to release ketoprofen at early stages after implantation. Morphology, size, in vitro release behavior, and in vivo biocompatibility were assessed. The effect of some preparation parameters was also evaluated. Polymeric microspheres were spherical and smooth, two populations of about 5 and $20 \mu \mathrm{m}$ of mean diameter characterized the particle size distribution.

Results: A high burst effect was observed for all preparations during in vitro release studies. Ketoprofen, plasticizing the polymeric matrix, could be responsible of this release behavior. Alginate/poly-Lornithine/alginate microcapsules were not modified upon ketoprofen-loaded microsphere encapsulation and an optimal dispersion was obtained. The composite system showed good biocompatibility when a high molecular weight polymer was employed. Therefore, a potentially suitable composite system for cell encapsulation was obtained.

Conclusions: This system may be successfully used to release non-steroidal anti-inflammatory drugs and other active molecules capable of improving cell system functional performance and lifespan.

During the past decades several encouraging advances have been achieved in pancreas transplantation for the treatment of patients with type 1 diabetes. Although pancreatic cell transplantation represents the most suitable treatment in selected patients, several side effects and problems limit the applicability of this procedure in a large number of patients. In fact, insulin independence in most patients is of relatively short duration, persisting on average for only 3-5 years [6]. Thus, new experimental approaches to significantly improve the lifespan of transplanted pancreatic endocrine cells are under investigation [7]. In the present study, composite microcapsules capable of releasing ketoprofen (a non-steroid anti-inflammatory drug) were used to encapsulate pancreatic endocrine cells. For this purpose, ketoprofen-loaded polylactic acid and polylactic-co-glycolic acid microspheres were prepared. This system may represent a potentially suitable composite system for transplantation of pancreatic islets that could improve the islet lifespan (minimizing the immunoreactivity to the graft). Further studies are obviously needed to evaluate the effectiveness of the composite system in human islet transplantation. 


\title{
The effect of glucose variability on the risk of microvascular complications in type 1 diabetes
}

\author{
Kilpatrick ES, Rigby AS, Atkin SL \\ Department of Clinical Biochemistry, Hull Royal Infirmary, Hull, UK \\ eric.kilpatrick@hey.nhs.uk
}

Diabetes Care 2006;29:1486-1490

Background: It is not known whether glycemic instability may confer a risk of microvascular complications that is in addition to that predicted by the mean blood glucose value alone. This study has analyzed data from the Diabetes Control and Complications Trial (DCCT) to assess the effect of glucose variability on the risk of retinopathy and nephropathy in patients with type 1 diabetes.

Methods: Pre- and postprandial 7-point glucose profiles were collected quarterly during the DCCT in 1,441 individuals. The mean area under the curve glucose and the SD of glucose variability within $24 \mathrm{~h}$ and between visits were compared with the risk of retinopathy and nephropathy, having adjusted for age, sex, disease duration, treatment group, prevention cohort, and phase of treatment.

Results: Multivariate Cox regression showed that within-day and between-day variability in blood glucose around a patient's mean value has no influence on the development or progression of either retinopathy $(\mathrm{p}=0.18$ and $\mathrm{p}=0.72$, respectively) or nephropathy $(\mathrm{p}=0.32$ and $\mathrm{p}=0.57)$. Neither preprandial $(\mathrm{p}=0.18)$ nor postprandial $(\mathrm{p}=0.31)$ glucose concentrations preferentially contribute to the probability of retinopathy.

Conclusions: This study has shown that blood glucose variability does not appear to be an additional factor in the development of microvascular complications. Also, pre- and postprandial glucose values are equally predictive of the small-vessel complications of type 1 diabetes.

The association between glycemic control defined as $\mathrm{HbA} 1 \mathrm{c}$ values and the risk of microvascular complications has clearly been defined in previous clinical trials (Stockholm Study, Oslo Study, Kroc Study and, finally, the DCCT) [8-11]. In fact, the higher the HbA1c values, the greater is the risk of either developing or worsening retinopathy, nephropathy and neuropathy. However, although $\mathrm{HbA1c}$ values have been shown to reflect the glycemic trend within the previous 3 months, this surrogate marker does not take into account the day-by-day and especially the 24-hour variability or to reveal hyperglycemic episodes [12]. Thus, despite low $\mathrm{HbA1c}$ levels, well-controlled patients may experience acute hyperglycemia which should increase the risk of diabetes-related complications [13]. Analyzing data from the 1,441 patients with type 1 diabetes recruited for the DCCT, this study evaluated the relationship between pre- or postprandial glycemia and the development of microvascular complications. It was shown that the development or progression of either retinopathy or nephropathy in patients with type 1 diabetes is not influenced by the variability in blood glucose. In fact, the risk of developing microvascular complications was not statistically different between patients with wide blood glucose fluctuations compared with those with little glycemic fluctuations throughout the day. Furthermore, the risk of developing microvascular complications was not related either to the pre- or to the postprandial glucose concentrations. Although these results should reassure patients and their caring physicians that no additional risk factors are added to glucose fluctuations, it appears of foremost importance to remark the clear relationship between glycemic control and microvascular complications. The current American Diabetes Association recommendations do not suggest a need to measure postprandial blood glucose in preference to basal values; this study confirms that there is no specific need for this to change in order to reduce the likelihood of microvascular complications. 


\section{Responses against islet antigens in NOD mice are prevented by tolerance to proinsulin but not IGRP}

Krishnamurthy B, Dudek NL, McKenzie MD, Purcell AW, Brooks AG, Gellert S, Colman PG, Harrison LC, Lew AM, Thomas HE, Kay TW

St Vincent's Institute, Fitzroy, Vic., Australia

eric.kilpatrick@hey.nhs.uk

J Clin Invest 2006;116:3258-3265

Background: Type 1 diabetes (T1D) is characterized by immune responses against several autoantigens expressed in pancreatic $\beta$ cells. T cells specific for proinsulin and islet-specific glucose-6-phosphatase catalytic subunit-related protein (IGRP) can induce T1D in NOD mice. However, whether immune responses to multiple autoantigens are caused by spreading from one to another or whether they develop independently of each other is unknown.

Methods and Results: As cytotoxic T cells specific for IGRP were not detected in transgenic NOD mice tolerant to proinsulin, the authors determined that immune responses against proinsulin are necessary for IGRP-specific T cells to develop. On the other hand, transgenic overexpression of IGRP resulted in loss of intra-islet IGRP-specific T cells but did not protect NOD mice from insulitis or T1D, providing direct evidence that the response against IGRP is downstream of the response to proinsulin.

Conclusions: These results suggest that pathogenic proinsulin-specific immunity in NOD mice subsequently spreads to other antigens such as IGRP.

Autoimmune response to a number of antigens represents the evolution of the course of type 1 diabetes in humans and NOD mice. The breakdown of normal tolerance to the $\beta$-cell allows the activation of $T$ cells to multiple specificities that results in a continuously increasing production of antibodies against the insulin-producing cells [1]. Although different antigens have been shown to be involved in the autoimmune destruction of $\beta$ cells, so far no convincing evidence has been reported to determine whether the disease is initiated by polyclonal activation of $T$ cells specific of single vs. multiple antigens. The present study investigated the relationship between immune response to proinsulin and other significant autoantigens. Evaluating the fate of IGRP $206-214^{-}$-specific T cells transgenic NOD mice overexpressing proinsulin 2 in APCs (NOD-PI mice), it was demonstrated that immune response to IGRP $_{206-214}$ does not develop in NOD-PI mice. Furthermore, using a NOD mice model overexpressing IGRP under the control of MHC class II promoter, it was shown that IGRP response occurs only when proinsulin responses have been established and that the immune response 'spreads' from proinsulin to IGRP. Although IGRP itself has not been shown to be a significant antigen in human type 1 diabetes, the concept of initiating and effector antigens may apply to it. These data suggest that the development of immune responses against multiple autoantigens is a necessary step. This is reminiscent of the clinical situation where individuals who are positive for a single autoantibody have a low risk of the disease, probably reflecting the lack of spreading of the immune response. This is an important distinction, because the effect of tolerance to proinsulin on immune responses to other antigens is a relevant issue in clinical trials for prevention of type 1 diabetes in humans. The findings of this study suggest that primary prevention should be most effective if targeted at the initiating antigen such as proinsulin. Once insulitis is established, tolerance induction to other $\beta$-cell antigens needs to be considered as well. Further investigations on how the response to autoantigens develops will help to fully understand how antigen-specific tolerance induction could prevent type 1 diabetes. 


\section{The relation of ambulatory blood pressure and pulse rate to retinopathy in type 1 diabetes mellitus: the renin-angiotensin system study}

Klein R, Moss SE, Sinaiko AR, Zinman B, Gardiner R, Suissa S, Donnelly SM, Kramer MS, Goodyer P, Strand T, Mauer M Department of Ophthalmology and Visual Sciences, University of Wisconsin School of Medicine and Public Health, Madison, Wisc., USA

kleinr@epi.ophth.wisc.edu

Ophthalmology 2006;113:2231-2236

Background: To examine the association of ambulatory blood pressure (ABP) and ambulatory pulse rate (APR) with diabetic retinopathy in persons with type 1 diabetes in the Renin-Angiotensin System Study (RASS), a multicenter primary diabetic nephropathy prevention trial.

Methods: Cross-sectional study of 194 normotensive RASS participants in three centers who are 16 years of age or older with type 1 diabetes mellitus of 2-20 years' duration. ABP and APR were monitored using standardized protocols. Patients were defined as non-dippers if the night-to-day ratios for both systolic and diastolic blood pressures were $>0.9$. Diabetic retinopathy was determined by masked grading of $30^{\circ}$ color stereoscopic fundus photographs of seven standard fields using the Early Treatment Diabetic Retinopathy Study severity scale.

Results: No diabetic retinopathy was present in 32\%, mild non-proliferative diabetic retinopathy (NPDR) was present in $55 \%$, and moderate to severe NPDR or proliferative diabetic retinopathy was present in $13 \%$ of the cohort. Neither 24-hour systolic ABP or diastolic ABP, daytime systolic or diastolic ABP, nor nighttime diastolic ABP were related to severity of diabetic retinopathy. Statistically significant associations were found between nighttime systolic ABP and mean ABP and diabetic retinopathy. Among those with no diabetic retinopathy, 19\% were non-dippers; for those with mild NPDR, $28 \%$ were non-dippers, and for those with severe NPDR or proliferative diabetic retinopathy, $36 \%$ were non-dippers $(\mathrm{p}=0.08)$. The ratio of nighttime to daytime APR, but not the 24-hour APR or daytime or nighttime APR, was related positively to the severity of DR. In multivariable analyses, only the nighttime systolic ABP was related to severity of diabetic retinopathy $(\mathrm{p}<0.05)$.

Conclusions: These data suggest that ABP, especially during the night, may provide a better measure than clinical $\mathrm{BP}$ regarding the relationship of $\mathrm{BP}$ to the severity of retinopathy in normotensive persons with type 1 diabetes without clinical diabetic nephropathy.

Diabetes retinopathy remains the major cause of acquired blindness in youngsters and adults of developed countries. Adequate screening programs allow to detect early diabetic retinopathy in a large proportion of young people with a disease duration of more than 11 years. Several studies evaluated the mechanisms involved in the onset and progression of early retinal alterations [11]. Although chronic hyperglycemia has been proven to be one of the foremost factors affecting retinal damage, other factors such us blood pressure have been supposed to play a role in diabetic retinopathy. By using data from the Renin-Angiotensin System Study, a parallel, double-blind, placebo-controlled, multicenter, primary prevention clinical trial of diabetic nephropathy [14], this study evaluated whether ABP and APR may be associated with the severity of diabetic retinopathy in normotensive patients with type 1 diabetes who have clinically normal renal function. It was clearly shown that nighttime systolic ABP is associated with the severity of diabetic retinopathy, while no association was detected between clinical blood pressure and clinical and APR. These results suggest that in normotensive patients with type 1 diabetes, the 24-hour continuous monitoring blood pressure could represent a marker of risk for diabetic retinopathy. Longitudinal studies are now needed to confirm the predictive role of ABP monitoring on later development of diabetic retinopathy. 


\section{Persistent renal hypertrophy and faster decline of glomerular filtration rate precede the development of microalbuminuria in type 1 diabetes}

Zerbini G, Bonfanti R, Meschi F, Bognetti E, Paesano PL, Gianolli L, Querques M, Maestroni A, Calori G, Del Maschio A, Fazio F, Luzi L, Chiumello G

Department of Medicine, San Raffaele Scientific Institute, Milan, Italy g.zerbini@hsr.it

Diabetes 2006;55:2620-2625

Background: Soon after the onset of type 1 diabetes, renal hypertrophy and hyperfiltration become manifest, particularly among patients who will subsequently develop diabetic nephropathy. Whether these early renal dysfunctions are involved in the pathogenesis of diabetic nephropathy is currently unclear. Methods: The authors evaluated, during the same day, kidney volume and glomerular filtration rate (GFR) in 146 patients with type 1 diabetes and normal renal function. All the individuals were then monitored for a mean of $9.5 \pm 4.4$ years for the development of microalbuminuria. Kidney volume and GFR were re-evaluated in a subset of 68 patients 4 years after baseline.

Results: During follow-up, microalbuminuria developed in 27 of 146 diabetic patients. At baseline, kidney volume $\left(312.8 \pm 52.6\right.$ vs. $281.4 \pm 46.1$ vs. $\left.236.8 \pm 41.6 \mathrm{ml} / 1.73 \mathrm{~m}^{2}, \mathrm{p}<0.05\right)$ but not GFR was increased in patients predisposed to microalbuminuria. The risk of progression was higher in patients with increased kidney volume $(\mathrm{p}=0.0058)$. Patients predisposed to microalbuminuria showed a stable increase in kidney volume $(p=0.003)$, along with a faster decline of GFR $(p=0.01)$. Persistent renal hypertrophy and faster decline of GFR precede the development of microalbuminuria in type 1 diabetes. Conclusions: These findings support the hypothesis that renal hypertrophy precedes hyperfiltration during the development of diabetic nephropathy.

Despite the development of microalbuminuria a few years after clinical onset of diabetes, it is well established that increased kidney volume and higher GFR may be detected as early as at onset of diabetes, especially in patients who will later develop nephropathy [15]. Although these abnormalities become evident quite simultaneously, the 'vascular hypothesis' retains that glomerular hyperfiltration due to defects of vascular control represents the primary event followed by hypertrophy [16]. On the other hand, the 'tubular hypothesis' suggests that the first event in diabetic nephropathy is increased kidney volume (due to hyperglycemia) followed by hyperfiltration [17]. This prospective study evaluated whether kidney volume and GFR are increased in subjects with type 1 diabetes mellitus and their relation to later development of microalbuminuria. In patients who developed microalbuminuria during the follow-up period, increased kidney volume was detected earlier than impaired GFR and both progressed during the course of diabetes. Furthermore, the rate of progression to microalbuminuria was significantly higher in patients with increased kidney volume. This study represents an important progress in the knowledge of diabetes-related kidney disease. A thorough understanding of the mechanisms involved in the development of renal hypertrophy is needed in order to evaluate approaches for the primary prevention of diabetic nephropathy.

\section{Lower bone mineral content in children with type 1 diabetes mellitus is linked to female sex, low insulin-like growth factor type I levels, and high insulin requirement}

Leger J, Marinovic D, Alberti C, Dorgeret S, Chevenne D, Marchal CL, Tubiana-Rufi N, Sebag G, Czernichow P Pediatric Endocrinology Unit and INSERM U 690, Hôpital Robert Debré, Paris, France

juliane.leger@rdb.ap-hop-paris.fr

J Clin Endocrinol Metab 2006;91:3947-3953

Background: Studies on bone mineral characteristics in children with type 1 diabetes have generated conflicting results. Our objective was to investigate bone mineral characteristics in children with type 1 diabetes and to analyze their associations with bone metabolism and the IGF-1 system.

Methods: A cohort of Caucasian patients with type 1 diabetes for at least 3 years and healthy children was recruited between January 2003 and June 2004. This was a university hospital-based study. A total of 
127 patients and 319 controls aged 6-20 years participated. Dual-energy x-ray absorptiometry was performed in patients and controls. Serum bone alkaline phosphatase, cross-laps, IGF-1, and IGF-binding protein 3 levels were determined in patients with values analyzed using normative data from 1,150 healthy children.

Results: After adjustment for age, sex, pubertal stage, and body mass index SD score, total body bone mineral content/lean body mass was significantly lower in patients than in controls $(\mathrm{p}<0.04)$. This difference was a result of the differences between the girls of the two groups. Girls with T1DM had significantly lower lumbar spine and total body bone mineral content than control girls $(\mathrm{p}=0.002)$, whereas no such difference was observed in boys. Serum bone alkaline phosphatase level was significantly lower in girls than in boys $(\mathrm{p}=0.04)$. Low serum IGF-1 levels and the administration of large amounts of insulin were found to have independent deleterious effects on bone mineral content for children of all ages and both sexes, whereas disease duration and glycosylated hemoglobin levels did not. Conclusions: A sex-related difference in the impairment of bone mineral characteristics was identified in children with T1DM. Longitudinal studies are required to investigate whether boys may gain slightly less bone mass during skeletal growth.

Due to systemic rather than portal injections of insulin, serum insulin concentrations are not physiological in patients with diabetes, leading to abnormalities of the GH-IGF-1 axis. Low hepatic insulin concentrations results in lower IGF-1 and IGFBP-3 levels that have been widely reported in patients with type 1 diabetes, particularly in those with poor metabolic control. The impaired GH-IGF-1 axis results in blunted growth and appears to influence bone mineralization [18]. Performing a dualenergy X-ray absorption, this cross-sectional study evaluated bone turnover, bone mineral content and body composition in a group of 127 young patients with type 1 diabetes at different pubertal stages. Similar values were observed in boys with type 1 diabetes and controls, whereas girls with type 1 diabetes had lower lumbar spine and total body bone mineral content and lower lean body mass. Furthermore, total body bone mineral content for lean body mass was lower in patients than controls. The observed differences resulted primarily from girls. There was a significant relation between insulin requirement and serum IGF-1-SDS. Although these preliminary data were obtained in a significant sample size, longitudinal data are needed in order to confirm bone alterations in young patients with type 1 diabetes. This could be relevant for the clinician. In fact, given the critical role of bone mass accretion during pubertal years, monitoring of bone mineral content in young patients with type 1 diabetes, especially girls, is recommended.

\section{Pre-type 1 diabetes dysmetabolism: maximal sensitivity achieved with both oral and intravenous glucose tolerance testing}

Barker JM, McFann K, Harrison LC, Fourlanos S, Krischer J, Cuthbertson D, Chase HP, Eisenbarth GS

Barbara Davis Center for Childhood Diabetes, University of Colorado Health, Sciences Center, Denver, Colo., USA jennifer.barker@uchsc.edu

J Pediatr 2007;150:31-36

Background: To determine the relationship of intravenous (IVGTT) and oral (OGTT) glucose tolerance test abnormalities to diabetes development in a high-risk prediabetic cohort and to identify an optimal testing strategy for detecting preclinical diabetes.

Methods: Diabetes Prevention Trial-Type 1 Diabetes (DPT-1) randomized subjects to oral $(\mathrm{n}=372)$ and parenteral $(\mathrm{n}=339)$ insulin prevention trials. Subjects were followed with IVGTTs and OGTTs. Factors associated with progression to diabetes were evaluated.

Results: Survival analysis revealed that higher quartiles of 2-hour glucose and lower quartiles of firstphase insulin response (FPIR) at baseline were associated with decreased diabetes-free survival. Cox proportional hazards modeling showed that baseline body mass index (BMI), FPIR, and 2-hour glucose levels were significantly associated with an increased hazard for diabetes. On testing performed within 6 months of diabetes diagnosis, 3\% (1/32) had normal FPIR and normal 2-hour glucose on OGTT. The sensitivities for impaired glucose tolerance (IGT) and low FPIR performed within 6 months of diabetes diagnosis were equivalent ( 76 vs. $73 \%$ ).

Conclusions: Most (97\%) subjects had abnormal IVGTTs and/or OGTTs before the development of diabetes. The highest sensitivity is achieved using both tests. 
Type 1 diabetes is a chronic autoimmune disease characterized by T-cell autoreactivity to pancreatic islets. $\beta$-Cell loss starts several months or years before the clinical onset of diabetes [1]. Thus, several early markers capable of reliably detecting patients at higher risk of diabetes are under investigation. In order to develop an optimal preclinical testing strategy to identify patients who eventually develop diabetes, the authors performed IVGTT and OGTT glucose tolerance tests in a high-risk prediabetic cohort participating in the Diabetes Prevention Trial-Type 1 Diabetes (DPT-1) [19]. In the present study, 258 subjects who developed diabetes and an additional 453 who were diabetes-free by the end of the study were followed with serial IVGTTs and OGTTs. Abnormalities of 2-hour glucose on OGTT and of first-phase insulin release during the IVGTT within 6 months before diabetes diagnosis were useful to identify individuals at high risk for clinical diabetes. Maximal sensitivity was achieved when both tests were used. First-phase insulin release $<10$ th percentile for age was not specific for diabetes and a significant proportion of the subjects who did not develop diabetes during a 2-year follow-up had an abnormal IVGTT. Furthermore, despite abnormalities of 2-hour glucose on OGTT, the majority of subjects maintained a normal fasting plasma glucose. A significant proportion of subjects had a normal OGTT (24\%) or IVGTT (22\%) in the 6 months before the onset of diabetes. A very small subset $(3 \%)$ of patients had normal results at both tests 6 months before diabetes onset. The large size and longitudinal follow-up provide important results for the interpretation of metabolic tests in antibody-positive relatives of patients with type 1 diabetes.

\section{Clinical trials, new treatments}

\section{Insulin detemir compared with NPH insulin in children and adolescents with type 1 diabetes}

Robertson KJ, Schoenle E, Gucev Z, Mordhorst L, Gall MA, Ludvigsson J

The Royal Hospital for Sick Children, Glasgow, UK

kjr@diabetes-scotland.org

Diabet Med 2007;24:27-34

Background: This study compared the effect of insulin detemir on glycemic control (HbA1c, fasting plasma glucose and variability thereof) with that of Neutral Protamine Hagedorn human isophane (NPH) insulin, both combined with insulin aspart, in children with type 1 diabetes mellitus, and compared the safety of these treatments.

Methods: In this 26-week, open-label, randomized (2:1), parallel-group study, 347 (140 prepubertal and 207 pubertal) children with type 1 diabetes, aged 6-17 years, received insulin detemir $(\mathrm{n}=232)$ or NPH insulin $(\mathrm{n}=115)$ once or twice daily, according to the prestudy regimen, plus premeal insulin aspart.

Results: The mean HbA1c decreased by approximately $0.8 \%$ with both treatments. After 26 weeks, the mean difference in HbA1c was $0.1 \%$ (95\% confidence interval $0.1-0.3$ ) (insulin detemir 8.0\%, NPH insulin $7.9 \%$ ). Within-subject variation in self-measured fasting plasma glucose was significantly lower with insulin detemir than with NPH insulin (SD 3.3 vs. 4.3, p $<0.001$ ), as was mean fasting plasma glucose $(8.4$ vs. $9.6 \mathrm{mmol} / \mathrm{l}, \mathrm{p}=0.022)$. The risk of nocturnal hypoglycemia was $26 \%$ lower with insulin detemir $(\mathrm{p}=0.041)$ and the risk of 24 -hour hypoglycemia was similar with the two treatments $(\mathrm{p}=0.351)$. The mean body mass index $(\mathrm{BMI}) \mathrm{z}$-score was lower with insulin detemir $(\mathrm{p}<0.001)$.

Conclusions: Basal-bolus treatment with insulin detemir or NPH insulin and premeal insulin aspart in children and adolescents with type 1 diabetes mellitus improved HbA1c to a similar degree. The lower and more predictable fasting plasma glucose, lower risk of nocturnal hypoglycemia and lower BMI observed with insulin detemir are clinically significant advantages compared with NPH insulin.

To date, basal bolus multiple insulin injections are recommended in young patients with type 1 diabetes in order to improve metabolic control and reduce the risk for microvascular complications [20]. During the past decade the importance of the 24-hour glycemic profile has stimulated the development of new basal insulin analogs (insulin glargine, insulin detemir) in order to reproduce the physiological serum insulin changes and obtain stable and predictable blood glucose levels [21]. The basal insulin analog detemir is an acylated derivative of human insulin which has been shown to result in a 
lower risk of hypoglycemia and in a predictable glycemic control with less day-to-day variation compared with NPH insulin in adult diabetic patients [22]. This multicenter open-label, randomized (2:1), parallel-group study in children with type 1 diabetes (140 prepubertal and 207 pubertal) investigated the efficacy and safety of detemir compared with NPH insulin. It was observed that transfer from $\mathrm{NPH}$ to detemir can be performed on a 1:1 insulin unit basis. Basal-bolus treatment for 26 weeks with insulin detemir plus premeal insulin aspart was associated with lower risk of nocturnal hypoglycemia but did not improve metabolic control. Although only few nocturnal time points are used in this study, insulin detemir could be useful in young patients with recurrent hypoglycemia. Furthermore, treatment with insulin detemir resulted in lower and more predictable fasting glucose levels. This could make titration towards more ambitious plasma glucose levels easier and safer, especially in children with a highly variable lifestyle. In conclusion, insulin detemir represents an encouraging new alternative for basal bolus therapy in children with diabetes, especially in those who experienced recurrent hypoglycemia or with a highly variable lifestyle. However, further studies are needed in children and adolescents.

\title{
Feasibility of automating insulin delivery for the treatment of type 1 diabetes
}

\author{
Steil GM, Rebrin K, Darwin C, Hariri F, Saad MF \\ Medtronic MiniMed, Northridge, Calif., USA \\ garry.steil@medtronic.com
}

Diabetes 2006;55:3344-3350

Background and Methods: An automated closed-loop insulin delivery system based on subcutaneous glucose sensing and subcutaneous insulin delivery was evaluated in 10 subjects with type 1 diabetes $(2$ men, 8 women, mean $( \pm S D)$ age $43.4 \pm 11.4$ years, duration of diabetes $18.2 \pm 13.5$ years). Closed-loop control was assessed over approximately $30 \mathrm{~h}$ and compared with open-loop control assessed over 3 days. Closed-loop insulin delivery was calculated using a model of the $\beta$-cell's multiphasic insulin response to glucose.

Results: Plasma glucose was $160 \pm 66 \mathrm{mg} / \mathrm{dl}$ at the start of closed loop and was thereafter reduced to $71 \pm 19$ by 1:00 p.m. (preprandial lunch). Fasting glucose the subsequent morning on closed loop was not different from target $(124 \pm 25$ vs. $120 \mathrm{mg} / \mathrm{dl}$, respectively; $\mathrm{p}>0.05)$. Mean glucose levels were not different between the open and closed loop $(133 \pm 63$ vs. $133 \pm 52 \mathrm{mg} / \mathrm{dl}$, respectively; $\mathrm{p}>0.65)$. However, glucose was within the range $70-180 \mathrm{mg} / \mathrm{dl} 75 \%$ of the time under closed loop vs. $63 \%$ for open loop. Incidence of biochemical hypoglycemia (blood glucose $<60 \mathrm{mg} / \mathrm{dl}$ ) was similar under the two treatments. There were no episodes of severe hypoglycemia.

Conclusions: The data provide proof of concept that glycemic control can be achieved by a completely automated external closed-loop insulin delivery system.

The clear association between metabolic control and risk of microvascular complications implies new methodological approaches to mimic physiological insulin variations and to obtain stable and predictable blood glucose levels around the clock [23]. Insulin pump delivery appears to be a suitable and practical method for the treatment of patients with type 1 diabetes. The recent availability of a realtime glucose sensor has raised the possibility of automated closed-loop systems capable of abolishing the operator-dependent link between glucose variations and insulin delivery. This study assessed a complex system comprising a real-time subcutaneous glucose sensor, an external insulin-delivery pump and a processor capable of reproducing the $\beta$-cell multiphasic glucose-induced insulin release. In 10 adult patients with diabetes, this closed-loop system was effective and able to achieve glucose levels similar to continuous subcutaneous insulin infusion with reduced variance. Furthermore, although no statistical differences were documented in terms of incidence of hypoglycemia, the system automatically suspended insulin delivery in all instances of hypoglycemia. However, postprandial levels were higher than in non-diabetic individuals. Although several improvements are needed, this is an important step ahead in the treatment of patients with type 1 diabetes. The availability of a closed-loop system which is able to integrate glucose levels automatically and translate them into insulin delivery should optimize glycemic control and reduce the risk of hypoglycemia, particularly during the night. 


\section{Longitudinal lipid screening and use of lipid-lowering medications in pediatric type 1 diabetes}

Maahs DM, Wadwa RP, McFann K, Nadeau K, Williams MR, Eckel RH, Klingensmith GJ

Barbara Davis Center for Childhood Diabetes and Department of Medicine, University of Colorado Health Sciences

Center, Aurora, Colo., USA

David.Maahs@uchsc.edu

J Pediatr 2007;150:146-150

Background: Because cardiovascular disease (CVD) is the leading cause of death in patients with type 1 diabetes, and dyslipidemia is an important CVD risk factor, the authors investigated dyslipidemia and its treatment in children with type 1 diabetes.

Methods: Subjects had type 1 diabetes $(\mathrm{n}=360)$, repeated lipid measurements $(\mathrm{n}=1,095$, mean $3.04 \pm 0.94$, range 2-11), and were seen between 1994 and 2004. Total cholesterol (TC), high-density lipoprotein cholesterol (HDL), and non-HDL cholesterol (non-HDL) were categorized on the basis of published guidelines. Age, diabetes duration, sex, body mass index, HbA1c, and lipid-lowering medication use were recorded. Predictors of TC, HDL, and non-HDL were determined.

Results: Sustained abnormalities existed for TC $\geq 200 \mathrm{mg} / \mathrm{dl}(16.9 \%)$; HDL $<35 \mathrm{mg} / \mathrm{dl}$ (3.3\%), and non$\mathrm{HDL} \geq 130 \mathrm{mg} / \mathrm{dl}(27.8 \%), \geq 160 \mathrm{mg} / \mathrm{dl}(10.6 \%)$, and $\geq 190 \mathrm{mg} / \mathrm{dl}(3.3 \%)$. Lipid-lowering medications were started on 23 patients. In mixed model longitudinal data analyses, HbA1c was significantly related to TC and non-HDL. Body mass index z-score was inversely related to HDL.

Conclusions: In this retrospective, longitudinal study of pediatric patients with type 1 diabetes with repeated lipid measurements, sustained abnormal levels for TC, HDL, and non-HDL were present. Prospective longitudinal data for dyslipidemia in youth with type 1 diabetes are needed.

The improvement in diabetes care has resulted in a decrease of microangiopathy and in a paradoxical increase of diabetes-related long-term angiopathy later in life. Several studies, including some in children and adolescents, have demonstrated a direct role of dyslipidemia in diabetes-related arterial wall changes [24]. However, despite the role of abnormal lipid contents as a cardiovascular risk factor, several diet or lifestyle intervention studies have failed to normalize lipid profiles. These results have supported the use of lipid-lowering medication in children and adolescents [25]. This retrospective longitudinal study evaluated a total of 1,095 lipid measurements and tested the effect and safety of lipid-lowering medications. The prevalence of abnormal lipid levels was elevated: $18.9 \%$ of patients had total cholesterol $\geq 200 \mathrm{mg} / \mathrm{dl}, 4.2 \%$ had HDL cholesterol $<35 \mathrm{mg} / \mathrm{dl}$. Non-HDL cholesterol was $\geq 130 \mathrm{mg} / \mathrm{dl}$ in $34.2 \%, \geq 160 \mathrm{mg} / \mathrm{dl}$ in $11.4 \%$, and $\geq 19.0 \mathrm{mg} / \mathrm{dl}$ in $5.8 \%$. Furthermore, higher total cholesterol levels were associated with a poor glycemic control and with older age. 23 of 360 patients who started lipid-lowering medication had a decrease of total and non-HDL cholesterol and an increase in HDL cholesterol; no significant side effects were reported. Although several limitations (inability to evaluate compliance with medications, differences in diet and lifestyle, differences in diabetes control, lipid profile evaluated in different laboratories), this study provides important information on lipid profiles in children and adolescents with diabetes. Further prospective longitudinal studies on the natural history of dyslipidemia and on the safety and efficacy of lipid-lowering medications are now needed. 


\section{TRPV1 + sensory neurons control $\beta$-cell stress and islet inflammation in autoimmune diabetes}

Razavi R, Chan Y, Afifiyan FN, Liu XJ, Wan X, Yantha J, Tsui H, Tang L, Tsai S, Santamaria P, Driver JP, Serreze D, Salter MW, Dosch HM

Neurosciences and Mental Health Program, The Hospital for Sick Children, Research Institute, University of Toronto, Toronto, Ont., Canada

hmdosch@sickkids.ca

Cell 2006;127:1123-1135

Background: In type 1 diabetes, T-cell-mediated death of pancreatic $\beta$ cells produces insulin deficiency. However, what attracts or restricts broadly autoreactive lymphocyte pools to the pancreas remains unclear.

Methods and Results: The authors report that transient receptor potential vanilloid-1+ pancreatic sensory neurons control islet inflammation and insulin resistance. Eliminating these neurons in diabetes-prone NOD mice prevents insulitis and diabetes, despite systemic persistence of pathogenic T-cell pools. Insulin resistance and $\beta$-cell stress of prediabetic NOD mice are prevented when transient receptor potential vanilloid-1+ neurons are eliminated. Transient receptor potential vanilloid-1 (NOD), localized to the Idd4.1 diabetes-risk locus, is a hypofunctional mutant, mediating depressed neurogenic inflammation. Delivering the neuropeptide substance P by intra-arterial injection into the NOD pancreas reverses abnormal insulin resistance, insulitis, and diabetes for weeks. Concordantly, insulin sensitivity is enhanced in trpv1 $1^{-1-}$ mice, whereas insulitis/diabetes-resistant NOD $\times$ B6Idd4-congenic mice, carrying wild-type transient receptor potential vanilloid-1, show restored transient receptor potential vanilloid-1 function and insulin sensitivity.

Conclusions: These data uncover a fundamental role for insulin-responsive transient receptor potential vanilloid-1+ sensory neurons in $\beta$-cell function and diabetes pathoetiology.

Type 1 diabetes in humans and NOD mice results from T-cell-mediated autoimmune destruction of insulin-producing $\beta$ cells [1]. The disease is initiated by polyclonal activation of T cells to multiple specific antigens caused by a breakdown in normal tolerance to the $\beta$ cell. Autoreactive T lymphocytes progressively infiltrate pancreatic islets of Langerhans reaching a complete and progressive destruction of $\beta$ cells and overt diabetes. Several theories have been proposed in order to explain the mechanism involved in the recruitment and activation of autoreactive T lymphocytes in mediating islet cell destruction. Recent studies have supposed a role of nervous system failure defined by the function of the primary sensory afferent neuron and immune system failure in autoimmune diseases [26]. In particular, a non-specific cation channel, the transient receptor potential vanilloid-1 protein expressed in sensory neurons, has been shown to play an important role in proinflammatory reactions. This study evaluated whether sensory neurons may have a role in type 1 diabetes. It was clearly shown that transient receptor potential vanilloid-1 sensory neurons appear critical for the immune-cell accumulation in the pancreas playing a pivotal role in the initiation and progression of islet inflammation mediated in part by the primary afferent neurons. This study represents the first evidence for a direct role of sensory neurons in islet physiology, opening new perspectives for therapeutic strategies to prevent progression of $\beta$ cell failure. 


\section{A genome-wide association study of non-synonymous SNPs identifies a type 1 diabetes locus in the interferon-induced helicase (IFIH1) region}

Smyth DJ, Cooper JD, Bailey R, Field S, Burren O, Smink LJ, Guja C, lonescu-Tirgoviste C, Widmer B, Dunger DB, Savage DA, Walker NM, Clayton DG, Todd JA

Juvenile Diabetes Research Foundation/Wellcome Trust Diabetes and Inflammation Laboratory, Cambridge Institute for Medical Research, Addenbrooke's Hospital, Cambridge, UK

john.todd@cimr.cam.ac.uk

Nat Genet 2006;38:617-619

Background and Methods: In this study the authors report convincing statistical support for a sixth type 1 diabetes (T1D) locus in the innate immunity viral RNA receptor gene region IFIH1 (also known as mda-5 or Helicard) on chromosome 2q24.3.

Results: The authors found the association in an interim analysis of a genome-wide non-synonymous SNP (nsSNP) scan, and validated it in a case-control collection and replicated it in an independent family collection. In 4,253 cases, 5,842 controls and 2,134 parent-child trio genotypes, the risk ratio for the minor allele of the nsSNP rs1990760 A $\rightarrow$ G (A946T) was $0.86(95 \%$ confidence interval $0.82-0.90)$ at $\mathrm{P}=1.42 \times 10^{-10}$.

Several epidemiological studies have focused the important role of genetic susceptibility on the development of type 1 diabetes. During the past decades, genetic analyses sustained by convincing and reproducible statistical support have identified five main type 1 diabetes susceptibility loci: the HLA class II genes, the insulin gene, the CTLA4 locus, PTPN22 and the relatively recently reported locus in the IL2RA/CD25 region on chromosome 10p15 [27-31]. Adopting a genome-wide nsSNP scan approach using a highly multiplexed, high-throughput molecular inversion probe (MIP) technology (Affymetrix), this study reports a new type 1 diabetes susceptibility locus in the chromosome 2q24.3 (risk ratio for the minor allele $\mathrm{G}=0.86 ; \mathrm{P}=1.42 \times 10^{10}$ ) in a total of 12,229 subjects (case, controls and parent-child). This locus is involved in the innate immunity viral RNA receptor gene region IFIH1. This gene is an early type I interferon (IFN) $\beta$-responsive gene, which may contribute to the apoptosis of virally infected cells in antiviral immune responses, thus making it a sensor or pathogen recognition receptor for viral infection. The large sample size based on large case-control and family collections and the convincing statistical analysis justifies further genetic analysis of the region in other populations, association analyses of other infectious and chronic diseases, and functional studies to assess if IFIH1 is the causal gene in the locus and A946T is the substitution responsible for the disease association. Furthermore, the genetic association between type 1 diabetes and IFIH1 could provide a molecular link between the development of diabetes and its previously reported associations with viral infections.

\section{An ATP-binding mutation (G334D) in KCNJ11 is associated with a sulfonylurea-insensitive form of developmental delay, epilepsy, and neonatal diabetes}

Masia R, Koster JC, Tumini S, Chiarelli F, Colombo C, Nichols CG, Barbetti F

Department of Cell Biology and Physiology, Washington University School of Medicine, St. Louis, Mo., USA

cnichols@wustl.edu

Diabetes 2007; 56:328-336

Background: Mutations in the pancreatic ATP-sensitive $\mathrm{K}^{+}$channel (K(ATP) channel) cause permanent neonatal diabetes mellitus (PNDM) in humans. All of the K(ATP) channel mutations examined result in decreased ATP inhibition, which in turn is predicted to suppress insulin secretion.

Methods and Results: Here the authors describe a patient with severe PNDM, which includes developmental delay and epilepsy, in addition to neonatal diabetes (developmental delay, epilepsy, and neonatal diabetes (DEND)), due to a G334D mutation in the Kir6.2 subunit of K(ATP) channel. The patient was wholly unresponsive to sulfonylurea therapy (up to $1.14 \mathrm{mg} \cdot \mathrm{kg}^{-1} \cdot \mathrm{day}^{-1}$ ) and remained insulin-dependent. Consistent with the putative role of G334 as an ATP-binding residue, reconstituted homomeric and 
mixed WT + G334D channels exhibit absent or reduced ATP sensitivity but normal gating behavior in the absence of ATP. In disagreement with the sulfonylurea insensitivity of the affected patient, the G334D mutation has no effect on the sulfonylurea inhibition of reconstituted channels in excised patches. However, in macroscopic rubidium-efflux assays in intact cells, reconstituted mutant channels do exhibit a decreased, but still present, sulfonylurea response.

Results: The results demonstrate that ATP-binding site mutations can indeed cause DEND and suggest the possibility that sulfonylurea insensitivity of such patients may be a secondary reflection of the presence of DEND rather than a simple reflection of the underlying molecular basis.

The continuously increasing knowledge on the genetic mechanism underlying neonatal diabetes mellitus has recently improved the treatment opportunity of both the permanent and transient form of neonatal diabetes mellitus. In particular, evidence for a direct and primary role of the pancreatic ATP-sensitive $\mathrm{K}$ channel $\left(\mathrm{K}_{\text {ATP }}\right)$ mutation in the development of permanent neonatal diabetes mellitus has increased the interest of sulfonylurea compounds as an alternative treatment to insulin injections in many patients with $\mathrm{K}_{\text {ATP }}$-induced diabetes [32]. However, treatment effectiveness and clinical features appear to be strongly related to the different mutations. In fact, convincing evidence exists for a genotype-phenotype relationship in which the severity of the mutation correlates with the severity of the disease. Ranging from mild to strong activating mutation of the $\mathrm{K}_{\text {ATP }}$, clinical features vary from transient neonatal diabetes mellitus with no neurological symptoms to a severe multisystemic pathology defined by developmental delay, epilepsy, and neonatal diabetes (DEND). In the present study a novel human ATP-binding mutation (G334D) in KCNJ11 (Kir6.2) associated with DEND was identified. Structurally, $K_{\text {ATP }}$ channels are hetero-octomers, consisting of four subunits each of the pore-forming Kir6.2 subunit (KCNJ11) and four regulatory sulfonylurea receptor subunits (SUR1, ABCC8). ATP inhibits the channel by directly interacting with Kir6.2 where binding of ATP at one of the four subunits is sufficient to close the channel. SUR1 mediates stimulation of channel activity by Mg-nucleotide as well as inhibition by sulfonylureas. The mutation described here determines absent or reduced ATP sensitivity, but normal gating behavior in the absence of ATP. This characteristic mutation appears to cause the unresponsiveness to sulfonylureas reported in this patient. This new mutation provides evidence of the tight relationship between genotype-phenotype-dependent clinical features and pharmacological susceptibility.

\section{New hormones}

\section{Adiponutrin gene is regulated by insulin and glucose in human adipose tissue}

Moldes M, Beauregard G, Faraj M, Peretti N, Ducluzeau PH, Laville M, Rabasa-Lhoret R, Vidal H, Clement K Department of Endocrinology, Cancer and Metabolism, Institut Cochin, Paris, INSERM, U567, Paris, France Eur J Endocrinol 2006;155:461-468

Background: Adiponutrin is a new transmembrane protein specifically expressed in adipose tissue. In obese subjects, short- or long-term calorie restriction diets were associated with a reduction in adiponutrin gene expression. Adiponutrin mRNA level was previously shown to be negatively correlated with fasting glucose plasma levels and associated with insulin sensitivity of non-diabetic obese and non-obese subjects. The purpose of the present work was to get more insight into the regulation of adiponutrin gene expression by insulin and/or glucose using clamp studies and to examine its potential dysregulation in subjects with a deterioration of glucose homeostasis.

Methods: Adiponutrin gene expression was quantified by reverse transcriptase-quantitative PCR in s.c. adipose tissue of healthy lean subjects after an euglycemic hyperinsulinemic clamp (EGHI), a hyperglycemic euinsulinemic clamp, and a hyperglycemic hyperinsulinemic (HGHI) clamp. Adiponutrin gene expression was also analyzed in patients with different levels of insulin resistance.

Results: During EGHI, insulin infusion induced adiponutrin gene expression 8.4-fold $(\mathrm{p}=0.008)$. Its expression was also induced by glucose infusion, although to a lesser extent (2.2-fold, $\mathrm{p}=0.03$ ). Infusion of both insulin and glucose (HGHI) had an additive effect on the adiponutrin expression 
(10-fold, $\mathrm{p}=0.008)$. In a pathological context, adiponutrin gene was highly expressed in the adipose tissue of type 1 diabetic patients with chronic hyperglycemia compared with healthy subjects. Conversely, adiponutrin gene expression was significantly reduced in type 2 diabetics $(p=0.01)$, but remained moderately regulated in these patients after the EGHI clamp (2.5-fold increase).

Conclusions: These results suggest a strong relationship between adiponutrin expression, insulin sensitivity, and glucose metabolism in human adipose tissue.

\begin{abstract}
Adipose tissue is now universally considered an endocrine tissue which is able to synthesize several endocrine and paracrine factors [33]. To date, several studies have documented their direct role in insulin sensitivity and metabolism. The metabolic influence of adipose-tissue-derived adipokines should play an important role on the metabolic state in patients with type 1 diabetes, especially during puberty. In fact, it is well known that puberty is characterized by insulin resistance and increased insulin requirement. This study evaluated the relationship between a novel adipocyte-expressed transmembrane protein (adiponutrin) and insulin sensitivity and glucose metabolism. A clamp study with different insulin and/or glucose infusion was performed in healthy males, in adults with type 1 diabetes and in patients with type 2 diabetes; adiponutrin gene expression and modulation was evaluated in subcutaneous adipose tissue. It was shown that adiponutrin gene expression is markedly stimulated by insulin and hyperglycemia, although less efficiency was shown in diabetic subjects. Insulin was shown to amplify the glucose-mediated gene expression. Furthermore, adiponutrin gene expression was increased in patients with type 1 diabetes, reduced in patients with type 2 diabetes and unmodified in obese subjects. Although the physiological role of adiponutrin in metabolism remains to be completely clarified, this study gives a relevant contribution in understanding adiponutrin effects. According to these data, a complete explanation of the metabolic effects of this new transmembrane protein is further needed in order to clarify its role in glucose homeostasis of patients with type 1 diabetes.
\end{abstract}

Reviews

\title{
Cardiovascular risk reduction in high-risk pediatric patients: a scientific statement from the American Heart Association Expert Panel on Population and Prevention Science; the Councils on Cardiovascular Disease in the Young, Epidemiology and Prevention, Nutrition, Physical Activity and Metabolism, High Blood Pressure Research, Cardiovascular Nursing, and the Kidney in Heart Disease, and the Interdisciplinary Working Group on Quality of Care and Outcomes Research: endorsed by the American Academy of Pediatrics
}

Kavey RE, Allada V, Daniels SR, Hayman LL, McCrindle BW, Newburger JW, Parekh RS, Steinberger J

National Heart, Lung, and Blood Institute, NIH, Bethesda, Md., USA

Circulation 2006;114:2710-2738

Background: Although for most children the process of atherosclerosis is subclinical, dramatically accelerated atherosclerosis occurs in some pediatric disease states, with clinical coronary events occurring in childhood and very early adult life. As with most scientific statements about children and the future risk for cardiovascular disease (CVD), there are no randomized trials documenting the effects of risk reduction on hard clinical outcomes. A growing body of literature, however, identifies the importance of premature CVD in the course of certain pediatric diagnoses and addresses the response to risk factor reduction.

Methods and Results: For this scientific statement, a panel of experts reviewed what is known about very premature CVD in 8 high-risk pediatric diagnoses and, from the science base, developed practical recommendations for management of cardiovascular risk.

Several studies have demonstrated a clear association between CVD risk markers and risk of developing micro- and macrovascular complications in young patients with type 1 diabetes. Although evident 
during adulthood, it is universally accepted that atherosclerosis begins early in life [34]. In fact, the exposure during childhood to diabetes-related metabolic alterations may induce changes in the arteries contributing to the development of atherosclerosis in adulthood. Several new markers have been associated with atherosclerotic vascular changes. Evaluating the published studies on premature CVD in 8 high-risk pediatric conditions, this scientific statement, endorsed by the American Academy of Pediatrics, provides relevant recommendations for the detection of cardiovascular risk in conditions like type 1 and type 2 diabetes or familial hypercholesterolemia.

\title{
Food for thought
}

\section{Effects of high-dose vitamin E supplementation on oxidative stress and microalbuminuria in young adult patients with childhood-onset type 1 diabetes mellitus}

\author{
Giannini C, Lombardo F, Curro F, Pomilio M, Bucciarelli T, Chiarelli F, Mohn A \\ Department of Pediatrics, University of Chieti, Italy \\ amohn@unich.it \\ Diabetes Metab Res Rev 2007 Feb [Epub ahead of print]
}

Background: The aim of this study was to evaluate the effects of high-dose vitamin E supplementation $(1,200 \mathrm{mg} /$ day) on reducing both microalbuminuria (MA) and oxidative stress in patients with type 1 diabetes mellitus (T1DM) and persistent MA.

Methods: The authors performed a 12-month, randomized, placebo-controlled, double-blind cross-over trial in 10 young Caucasian adults $(7 \mathrm{~m} / 3 \mathrm{f}$; mean age $18.87 \pm 2.91$ years $)$ with T1DM and persistent MA. At baseline and at the end of the treatment period, determination of albumin excretion rate (AER) and $\mathrm{HbA} 1 \mathrm{c}$ and evaluation of the oxidant/antioxidant status were performed.

Results: At the beginning of the study, AER and HbA1c were not significantly different between the vitamin $\mathrm{E}$ and placebo group. No differences in terms of oxidant and antioxidant status were found between the two groups. This was associated with no significantly different urinary VEGF and TGF- $\beta$ levels. After 6 months, no significant differences in AER were observed between the two groups $(\mathrm{p}=0.59)$. However, plasma and LDL-vitamin $\mathrm{E}$ content were significantly higher in the vitamin $\mathrm{E}$ group compared to the placebo group $(\mathrm{p}=0.0001$ and $\mathrm{p}=0.004$, respectively). This was associated with a significantly longer lag phase $(\mathrm{p}=0.002)$ and lower MDA $(\mathrm{p}=0.049)$. However, no statistically significant differences were detected in terms of VEGF and TGF- $\beta$ urinary levels.

Conclusions: These data demonstrate that high-dose vitamin E supplementation reduces markers of oxidative stress and improves antioxidant defense in young patients with T1DM. However, although it positively affects the oxidant/antioxidant status, vitamin E supplementation does not reduce AER in patients with T1DM and persistent MA.

Previous studies have clarified the role of chronic hyperglycemia on microvascular complications showing a direct positive correlation between poor metabolic control and the development of diabetes complications [11]. However, microvascular complications may also occur in some patients with satisfactory glycemic control; these observations have paved the way for better studying the mechanisms involved in endothelial dysfunction in order to provide new approaches to prevent or reduce microvascular damage in patients with type 1 diabetes. The present study investigated whether microalbuminuria and oxidative stress could be affected by high-dose vitamin E supplementation $(1,200 \mathrm{mg} /$ day $)$ given orally, in a group of young adult patients with childhood-onset type 1 diabetes and persistent microalbuminuria. This is the first randomized, placebo-controlled, double-blind crossover trial with vitamin $\mathrm{E}$ in young adult Caucasian patients with type 1 diabetes. Although the small sample size population is a major flaw of this study, it is clinically relevant that orally supplemented high-dose vitamin $\mathrm{E}$ has no effects on glomerular functional changes in patients with type 1 diabetes and persistent microalbuminuria. During the 6-month period, no significant changes in albumin excretion rate occurred. In the past decades, the pathophysiological course and anatomic changes which occur in diabetic kidney have been characterized, ruling out the importance role of microalbuminuria 
on predicting later overt diabetic nephropathy. Furthermore, several studies have shown that antioxidant treatment might reduce heart disease morbidity and mortality in the general population. It is well known that chronic hyperglycemia causes alteration in the oxidant/antioxidant status in patients with type 1 diabetes, especially in those with overt vascular complications. However, the lack of effects on albumin excretion rate in patients enrolled in this study show that antioxidant treatment allows a significant improvement in the oxidant-antioxidant status without affecting the established functional glomerular abnormalities. All patients enrolled in this study had persistent microalbuminuria, therefore limiting the conclusions of this study to this specific group of patients. Further studies are therefore needed to evaluate the effect of antioxidant treatment in patients with type 1 diabetes.

References

1. Tisch R, McDevitt H: Insulin-dependent diabetes mellitus. Cell 1996;85:291-297.

2. Rus H, Pardo CA, Hu L, Darrah E, Cudrici C, Niculescu T, et al: The voltage-gated potassium channel Kv1.3 is highly expressed on inflammatory infiltrates in multiple sclerosis brain. Proc Natl Acad Sci USA 2005;102:11094-11099.

3. Ryan EA, Bigam D, Shapiro AM: Current indications for pancreas or islet transplant. Diabetes Obes Metab 2006;8:1-7.

4. Tam PP, Williams EA, Chan WY: Gastrulation in the mouse embryo: ultrastructural and molecular aspects of germ layer morphogenesis. Microsc Res Tech 1993;26:301-328.

5. Liew CG, Moore H, Ruban L, Shah N, Cosgrove K, Dunne M, et al: Human embryonic stem cells: possibilities for human cell transplantation. Ann Med 2005;37:521-532.

6. Rother KI, Harlan DM: Challenges facing islet transplantation for the treatment of type 1 diabetes mellitus. J Clin Invest 2004;114:877-883.

7. Calafiore R: Alginate microcapsules for pancreatic islet cell graft immunoprotection: struggle and progress towards the final cure for type 1 diabetes mellitus. Expert Opin Biol Ther 2003;3:201-205.

8. Reichard P, Britz A, Carlsson P, Cars I, Lindblad L, Nilsson BY, et al: Metabolic control and complications over 3 years in patients with insulin-dependent diabetes (IDDM): the Stockholm Diabetes Intervention Study (SDIS). J Intern Med 1990;228:511-517.

9. Bangstad HJ, Osterby R, Dahl-Jorgensen K, Berg KJ, Hartmann A, Hanssen KF: Improvement of blood glucose control in IDDM patients retards the progression of morphological changes in early diabetic nephropathy. Diabetologia 1994;37:483-490.

10. Home PD, Alberti KG, Rodger NW, Burrin JM: Conference on insulin pump therapy in diabetes. Multicenter study of effect on microvascular disease. The Central Biochemistry Laboratory in the Multicenter Kroc Study. Problems and proposals. Diabetes 1985;34(suppl 3):17-21.

11. Diabetes Control and Complications Trial Research Group: The effect of intensive treatment of diabetes on the development and progression of long-term complications in insulin-dependent diabetes mellitus. N Engl J Med 1993;329: 977-986.

12. Hirsch IB, Brownlee M: Should minimal blood glucose variability become the gold standard of glycemic control? J Diabetes Complications 2005;19:178-181.

13. American Diabetes Association: Postprandial blood glucose. Diabetes Care 2001;24:775-778.

14. Mauer M, Zinman B, Gardiner R, Drummond KN, Suissa S, Donnelly SM, et al: ACE-I and ARBs in early diabetic nephropathy. J Renin Angiotensin Aldosterone Syst 2002;3:262-269.

15. Amin R, Turner C, van Aken S, Bahu TK, Watts A, Lindsell DR, et al: The relationship between microalbuminuria and glomerular filtration rate in young type 1 diabetic subjects: the Oxford Regional Prospective Study. Kidney Int 2005;68: 1740-1749.

16. O’Bryan GT, Hostetter TH: The renal hemodynamic basis of diabetic nephropathy. Semin Nephrol 1997;17:93-100.

17. Bak M, Thomsen K, Christiansen T, Flyvbjerg A: Renal enlargement precedes renal hyperfiltration in early experimental diabetes in rats. J Am Soc Nephrol 2000;11:1287-1292.

18. Dunger DB, Acerini CL: IGF-1 and diabetes in adolescence. Diabetes Metab 1998;24:101-107.

19. Skyler JS, Krischer JP, Wolfsdorf J, Cowie C, Palmer JP, Greenbaum C, et al: Effects of oral insulin in relatives of patients with type 1 diabetes: The Diabetes Prevention Trial - Type 1. Diabetes Care 2005;28:1068-1076.

20. Standards of medical care in diabetes - 2006. Diabetes Care 2006;29(suppl 1):S4-S42.

21. Heise T, Nosek L, Ronn BB, Endahl L, Heinemann L, Kapitza C, et al: Lower within-subject variability of insulin detemir in comparison to NPH insulin and insulin glargine in people with type 1 diabetes. Diabetes 2004;53:1614-1620.

22. Vague P, Selam JL, Skeie S, De Leeuw I, Elte JW, Haahr H, et al: Insulin detemir is associated with more predictable glycemic control and reduced risk of hypoglycemia than NPH insulin in patients with type 1 diabetes on a basal-bolus regimen with premeal insulin aspart. Diabetes Care 2003;26:590-596.

23. Hovorka R: Continuous glucose monitoring and closed-loop systems. Diabet Med 2006;23:1-12.

24. Soedamah-Muthu SS, Chaturvedi N, Toeller M, Ferriss B, Reboldi P, Michel G, et al: Risk factors for coronary heart disease in type 1 diabetic patients in Europe: the EURODIAB Prospective Complications Study. Diabetes Care 2004;27: 530-537.

25. Management of dyslipidemia in children and adolescents with diabetes. Diabetes Care 2003;26:2194-2197.

26. Carrillo J, Puertas MC, Alba A, Ampudia RM, Pastor X, Planas R, et al: Islet-infiltrating B-cells in nonobese diabetic mice predominantly target nervous system elements. Diabetes 2005;54:69-77.

27. Cucca F, Lampis R, Congia M, Angius E, Nutland S, Bain SC, et al: A correlation between the relative predisposition of MHC class II alleles to type 1 diabetes and the structure of their proteins. Hum Mol Genet 2001;10:2025-2037.

28. Barratt BJ, Payne F, Lowe CE, Hermann R, Healy BC, Harold D, et al: Remapping the insulin gene/IDDM2 locus in type 1 diabetes. Diabetes 2004;53:1884-1889.

29. Ueda H, Howson JM, Esposito L, Heward J, Snook H, Chamberlain G, et al: Association of the T-cell regulatory gene CTLA4 with susceptibility to autoimmune disease. Nature 2003;423:506-511.

30. Smyth D, Cooper JD, Collins JE, Heward JM, Franklyn JA, Howson JM, et al: Replication of an association between the lymphoid tyrosine phosphatase locus (LYP/PTPN22) with type 1 diabetes, and evidence for its role as a general autoimmunity locus. Diabetes 2004;53:3020-3023. 
31. Vella A, Cooper JD, Lowe CE, Walker N, Nutland S, Widmer B, et al: Localization of a type 1 diabetes locus in the IL2RA/CD25 region by use of tag single-nucleotide polymorphisms. Am J Hum Genet 2005;76:773-779.

32. Pearson ER, Flechtner I, Njolstad PR, Malecki MT, Flanagan SE, Larkin B, et al: Switching from insulin to oral sulfonylureas in patients with diabetes due to Kir6.2 mutations. N Engl J Med 2006;355:467-477.

33. Trayhurn P, Wood IS: Adipokines: inflammation and the pleiotropic role of white adipose tissue. Br J Nutr 2004;92: 347-355.

34. Kavey RE, Daniels SR, Lauer RM, Atkins DL, Hayman LL, Taubert K: American Heart Association guidelines for primary prevention of atherosclerotic cardiovascular disease beginning in childhood. Circulation 2003;107:1562-1566. 
